FULL TEXT ARTICLE

POSITION PAPER

\title{
Willingness of European healthcare workers to undergo vaccination against seasonal influenza: current situation and suggestions for improvement
}

\section{George Kassianos}

President, British Global and Travel Health Association, Wokingham, Berkshire, UK

\section{Citation}

Kassianos G. Willingness of European healthcare workers to undergo vaccination against seasonal influenza: current situation and suggestions for improvement. Drugs in Context 2015; 4: 212268. doi: 10.7573/dic. 212268

\section{Copyright}

Copyright @ 2015 Kassianos G. Distributed under the terms of the Creative Commons License Deed CC BY NC ND 3.0 which allows anyone to copy, distribute, and transmit the article provided it is properly attributed in the manner specified below. No other uses without permission.

\section{Correct attribution}

Copyright (c) 2015 Kassianos G. http://dx.doi.org/10.7573/ dic.212268. Published by Drugs in Context under Creative Commons Attributions License Deed CC BY NC ND 3.0.

\section{Article URL}

http://www.drugsincontext.com/willingness-europeanhealthcare-workers-undergo-vaccination-seasonalinfluenza-current-situation-suggestions-improvement

\section{Correspondence}

George Kassianos, FRCGP, FBHS, FESC, FBGTHA, FAcadMed, FFTM RCPS Glasg, President, British Global and Travel Health Association and Clinical Immunisation Lead, Royal College of General Practitioners, 61 Plough Lane, Wokingham, Berkshire, RG40 1RQ, UK.gckassianos@btinternet.com

\section{Provenance}

Submitted, externally peer reviewed

\section{Dates}

Submitted: 25 November 2014

Accepted for publication, subject to peer review: 25 November 2014

Revised manuscript submitted: 18 December 2014

Accepted for publication: 19 December 2014

Publication date: 21 January 2015

\section{Publisher \& contact information}

Drugs in Context is published by Just Medical Media Ltd, Undermount, Rydal, Ambleside, Cumbria, LA22 9LT, UK; ISSN 1740-4398; Just Medical Media Limited is registered in England Number 6891187; VAT GB 945171322

\section{Julia Savory}

Head of Digital Publishing and Submissions Management julia@justmedicalmedia.com; Tel: +44 (0)1242910999

\section{Abbreviations}

EU, European Union; HCWs, healthcare workers 


\section{Specialist Editor-in-Chief}

\section{Professor Thomas D Szucs, MD}

Professor in Pharmaceutical Medicine and Director of ECPM at the University of Basel, Switzerland

\section{Specialist editorial board members}

\section{Dr Patricia R Blank, MPH}

Senior Research Scientist, ECPM Institute of Pharmaceutical Medicine, University of Basel, Switzerland

\section{Dr George Kassianos, FRCGP, FBHS, FESC, FBGTHA, FAcadMEd, FFTM RCPSGlasg}

General Practitioner, Bracknell, Berkshire, UK; President, British Global \& Travel Health Association and Clinical Immunisation Lead, Royal College of General Practitioners; Fellow of the European Society of Cardiology

\section{Professor Jennifer Robinson, MD}

Clinical Assistant Professor, Department of Pharmacotherapy, Washington State University College of Pharmacy, Spokane, WA, USA

\section{Group Editor-in-Chief}

\section{Christopher Blanchette, PhD, MBA}

Associate Dean for Research and Research Associate Professor in the Department of Public Health Sciences at the University of North Carolina and Director of Health Economics \& Outcomes Research at Otsuka America Pharmaceutical Inc, USA

\section{Expert Advisers - Epidemiology and biostatistics}

\section{Alex K Exuzides, PhD}

Director, ICON Clinical Research Inc, California, USA

\section{Professor Scott L Friedman, MD}

Fishberg Professor of Medicine, Dean for Therapeutic Discovery Chief, Division of Liver Diseases, Mount Sinai School of Medicine, New York, USA

\section{Carl De Moor, PhD}

Senior Principal, Epidemiology and Leader Epidemiology, Safety and Risk Management Center of Excellence Americas, IMS Health Inc, USA

\section{Dr John H Walker, OCT, MBA, PhD}

Professor, Goodman School of Business, Brock University, St Catharines, Ontario, Canada

To see the full Drugs in Context Editorial Board, please visit www.drugsincontext.com/editorial-board

\section{Expert Adviser - Publication Ethics}

\section{Dr Elizabeth (Liz) Wager}

Publications Consultant, Princes Risborough, UK; Visiting Professor, University of Split School of Medicine, Croatia; Former Chair (2009-2012), Committee on Publication Ethics (COPE)

\section{Editor-in-Chief Emeritus \\ Dr George Kassianos, FRCGP, FBHS, FESC, FBGTHA, FAcadMEd, FFTM RCPSGlasg}

General Practitioner, Bracknell, Berkshire, UK; President British Global \& Travel Health Association and Clinical Immunisation Lead, Royal College of General Practitioners; Fellow of the European Society of Cardiology

\section{Specialist Advisor - Clinical Pharmacology}

Dr Richard White, MA, PhD

Consulting Partner and Director, Oxford PharmaGenesis Ltd, UK 


\section{Willingness of European healthcare workers to undergo vaccination against seasonal influenza: current situation and suggestions for improvement}

George Kassianos

President, British Global Travel and Health Association and Clinical Immunisation Lead, Royal College of General Practitioners, Wokingham, Berkshire, UK

\section{Citation}

Kassianos G. Willingness of European healthcare workers to undergo vaccination against seasonal influenza: current situation and suggestions for improvement. Drugs in Context 2015; 4: 212268. doi: 10.7573/dic.212268

\begin{abstract}
Uptake of vaccination against seasonal influenza in healthcare workers (HCWs) is, in general, low (vaccine coverage of 6-54\%), as is awareness of its importance, and has been decreasing in most European Union (EU) countries in recent years. By virtue of their working environment, HCWs are at an increased risk of influenza infection and of subsequently transmitting the virus to vulnerable patients, in whom disease burden is significant. It could be argued that a similar or higher target vaccination rate
\end{abstract}

\section{Introduction}

Vaccination against seasonal influenza is recommended in healthcare workers (HCWs). In the European Union (EU), HCWs are defined as: clinicians; midwives; community-based, practice, and hospital-based nurses; paramedics, occupational therapists; physiotherapists; radiographers; care-based social workers; community and hospital-based pharmacists; students, trainees, and administrative staff based in a clinical setting. However, the level of uptake is low $(6-54 \%)[1,2]$ and vaccination recommendations vary between countries in terms of strength and specificity [1,3-6]. Coverage in the United Kingdom (UK) is one of the highest in the EU, with recent data in England showing vaccine uptake by frontline HCWs of $46 \%$ and $55 \%$ in the $2012 / 13$ and $2013 / 14$ influenza seasons, respectively [7]. However, in France, Spain, Germany and Italy, the results from a survey carried out in 2011 using qualitative data from face-to-face interviews showed a widespread lack of awareness and understanding among HCWs regarding the importance of vaccination against influenza, which may (at least in part) explain the low level of vaccine uptake [8]. to that recommended for older age groups and people with chronic medical conditions (75\%) should be applied to HCWs, and the European Council recommends Member States to improve vaccination coverage in this population. In this context, better education of HCWs is needed to increase awareness and highlight the importance of HCW vaccination for the benefit of public health, particularly for their patients, who may be at risk of serious complications that could lead to disability or death. Secondary to these professional responsibilities, personal benefits (as well as benefits to close family and friends) should also be emphasised. Misconceptions that create barriers to vaccination need to be discussed openly and objections placed in the context of public health.

Keywords: influenza, healthcare worker, vaccination, Europe, awareness, disease burden

Seasonal influenza is an acute viral infection with an incubation time from infection to symptoms of $\approx 1-2$ days. It is characterized by intense symptoms of high fever, pain in muscles and joints, fatigue and, often, a non-productive cough. While symptomatically similar in some respects to the common cold (e.g., cough, runny nose, headache), influenza infection is associated with fever (usually $30-40^{\circ} \mathrm{C}$ ) and can result in symptoms that develop much quicker and with much greater intensity and can last 1-2 weeks. The common cold does not, in general, result in serious health problems. However, influenza infection, despite a common misconception of not being severe or life-threatening, can lead to pneumonia, secondary bacterial infections, hospitalisation and, occasionally, death, particularly in certain risk groups [9]. Those at particular risk of developing severe or life-threatening sequelae are: pregnant women; children aged 6 months to 5 years; the elderly ( $\geq 65$ years of age); those with chronic medical conditions [2]. The European Council recommends a target vaccination rate to Member States of $75 \%$ in the elderly and, if possible, for all people aged $>6$ months with chronic medical conditions $[1,2,10]$. 
HCWs are, in general, healthy adults. Hence, they are not at high risk of serious complications after influenza infection. However, they do comprise a recommended target group for vaccination against seasonal influenza [11]. This recommendation is based on the increased exposure of HCWs to the viruses of seasonal influenza by virtue of their working environment, and hence the increased risk of HCWs transmitting the infection, which could be particularly relevant for patients at risk of serious consequences of infection. During hospitalisation, patients have 5-35-times greater risk of acquiring influenza or an influenza-like illness if exposed to infected patients or HCWs [12]. When assessed within long-term care settings, the benefits of vaccination against influenza, with efficacy of $70-90 \%$, to HCWs have been estimated to be a reduction in days of sick leave of $53 \%$ $[13,14]$. For patients, the benefits have been estimated to be a reduction in deaths among the elderly of $44 \%$, and a reduction in cases of influenza-like illness of $43 \%[3,15]$.

Greater awareness of HCWs with regard to their professional responsibility to be vaccinated to reduce the risk of transmission of influenza (and therefore reduce the risk of severe disease in at-risk patients with whom they are in frequent contact) should help to increase rates of vaccine uptake in HCWs and in the population as a whole through better advocacy. A target vaccination rate for HCWs is lacking, but the European Council recommends Member States to improve vaccination coverage in this population. A coverage rate of $75 \%$ defined for at-risk populations could be used as a benchmark for HCWs. It could be argued that an even higher rate should be recommended for HCWs because the logistics of vaccination of HCWs are much more straightforward than those for accessing at-risk populations. A suggestion, therefore, would be to make influenza vaccination of HCWs the rule rather than the exception. Ideally, all HCWs without contraindications to influenza vaccination and who have direct contact with patients should be vaccinated. The target rate of $75 \%$ defined for at-risk individuals could be used as an initial milestone towards achievement of $100 \%$ vaccination of non-contraindicated HCWs.

In Europe (which used to be the vaccine coverage standard that other global regions aimed to emulate), vaccination rates against seasonal influenza are, in general, declining (with exceptions such as the UK and the Netherlands). Simultaneously, vaccination rates are increasing in other parts of the world as vaccination programmes are initiated [1]. A target of influenza vaccination coverage in at-risk groups in the $\mathrm{EU}$ of $75 \%$ is considered to be pragmatic and achievable. However, vaccination rates are below this target, and only half of the preventable deaths due to complications arising from influenza infection are currently being avoided in the EU [16]. The influenza virus can transmit readily to at-risk individuals even before symptoms are manifested, so vaccination of HCWs is particularly important.
In this article, the burden of seasonal influenza and the options to combat it are considered briefly. However, the main focus is evaluation of the drivers and barriers for vaccine uptake in the EU by HCWs.

\section{Disease burden}

Assuming that $\approx 5-20 \%$ of the EU population (based on an extrapolation from US estimates [17]) are affected by seasonal influenza each year, this percentage equates to $\approx 25-100$ million individuals who are infected each year. Those at risk of serious complications if infected, together with HCWs, comprise $\approx 36 \%$ of the EU population ( $\approx 180$ million individuals) $[9,11,16,18,19]$. Worldwide, $\leq 90 \%$ of influenza-related deaths occur in those aged $>65$ years, and $\approx 38,500$ influenza-related deaths occur each year in Europe [19].

Due to their regular contact with patients, HCWs may be at increased risk of contracting influenza [20]. However, being mostly healthy adults of working age, HCWs do not comprise a group that, as a whole, is particularly at risk of serious complications (e.g. infants, elderly). Their contact with vulnerable and ill patients, however, represents an increased risk of HCWs transmitting the infection towards those at risk of serious complications.

As well as deaths and serious complications requiring care, absenteeism and reduced productivity contribute to an economic burden to the EU of €6-14 billion per year [16]. Table 1 presents the estimated benefits from current vaccination coverage rates if this coverage were to be increased to $75 \%$ in recommended target populations.

\section{Options for prevention}

\section{Vaccination}

Vaccination is recognised by the World Health Organisation, European Centre for Disease Prevention and Control, and the UK National Institute for Health and Care Excellence as the most effective approach for the prevention of seasonal influenza. Vaccination is documented as the first-line intervention employed to control the impact of seasonal influenza on public health, and to minimise the serious complications that can arise in at-risk populations [21,22]. In Member States of the EU, concerted action is recommended to encourage vaccination among those at risk of serious complications to ensure a target coverage rate of $75 \%[1,2]$.

\section{Other preventative options}

Other than vaccination, options to prevent infection (or to minimise its spread if infection has occurred) include: avoiding close contact with infected individuals; limiting contact with others if infected; frequent (and effective) hand washing; 
Table 1. Estimated public-health and economic benefits from vaccination against seasonal influenza at current levels and $75 \%$ vaccination coverage in the European Union.

\section{Influenza-related consequence}

\begin{tabular}{ll} 
& $\begin{array}{l}\text { avoided if vaccination rate increased } \\
\text { to } \mathbf{7 5 \%} \text { (recommended target) }\end{array}$ \\
\hline Number of patients & $1.6-1.7$ million \\
\hline Doctor visits & $680,000-770,000$ \\
\hline Hospitalisations & $24,000-31,000$ \\
\hline Lost work days & $880,000-1,000,000$ \\
\hline Total costs & - \\
\hline Deaths & $10,000-14,000$
\end{tabular}

\begin{tabular}{l}
$\begin{array}{l}\text { Estimate of additional cost avoided } \\
\text { if vaccination rate increased to } 75 \% \\
\text { (recommended target) }\end{array}$ \\
\hline - \\
$€ 20-23$ million \\
$€ 57-75$ million \\
$€ 112-128$ million \\
$€ 190-226$ million \\
\hline
\end{tabular}
$-$

Adapted from Preaud et al., 2014 [16].

avoiding touching the eyes/nose/mouth of infected individuals; covering the mouth/nose if sneezing, together with use and appropriate disposal of paper tissue; use of face-masks; staying at home if infected [23]. In addition, drugs such as oseltamivir, amantadine, or zanamivir can be used in a prophylactic manner to limit the spread of infection. However, in the EU, it is recommended that the availability and use of these anti-viral agents should not be an excuse to reduce efforts to provide influenza vaccination [22,24].

Overall, the measures described above do not compare with the importance and effectiveness of a well-implemented vaccination programme to minimise the spread of infection and symptoms. Vaccination is the most effective way to prevent the occurrence and spread of influenza and the measures outlined above are very much second line in the battle to control seasonal influenza.

\section{Current situation regarding vaccination uptake in HCWs}

In general, there is a perception among HCWs in the EU that vaccination against seasonal influenza is the most effective means of prevention of infection. Currently, all Member States of the EU recommend vaccination of HCWs, which is, in general, provided free of charge to maximise health benefits to the public and reduce disease burden. Nevertheless, vaccination rates of HCWs in the EU remain low, with most considering vaccination to be more important for their older, frailer patients than for themselves [8]. Countries that monitor vaccination coverage of HCWs have reported a very low rate [1]. There is a low perception of risk in HCWs, particularly with regard to complications after influenza infection, and a belief that HCWs will have been immunised naturally through contact with patients suffering from influenza [8]. The misconception that influenza is not a potentially serious disease is not uncommon among HCWs. Also, there is low awareness among many HCWs of their being at risk of infection or of the passthrough benefits of vaccination to patients as well as to close family and friends. Furthermore, some HCWs have raised concerns regarding becoming infected by the vaccine itself and cite scepticism towards vaccination per se. Other HCWs have stated that they consider vaccination to be inconvenient and time-consuming, or have concerns regarding the possible side-effects of vaccination. Taken together, these factors represent significant barriers to adequate vaccination coverage of HCWs in the EU [2].

HCWs must be reminded of their at-risk status regarding influenza infection, and of the necessity for vaccination to protect those that may be in their care and at risk of serious complications if they have been infected. Such a mind-set of professional responsibility is not currently the norm in HCWs in the EU, and needs to be addressed.

As well as reducing the risk of infection in vulnerable patients, a secondary benefit of convincing HCWs to be vaccinated against seasonal influenza is a likely positive effect on patient acceptance of vaccination. In this way, better informed HCWs can recommend vaccination more strongly to all recommended target groups among their patients.

\section{Raising awareness}

In a qualitative survey in 2011 in France, Germany, Italy and Spain using in-depth interviews of physicians and nurses [8], the main message reported by interviewees of the campaign for vaccination against seasonal influenza was that 'the flu vaccine has arrived'. Such a simplistic approach needs to be improved significantly by the education of HCWs about the importance of influenza vaccination, particularly in the context of minimising transmission to their vulnerable patients. More personalised information regarding the severity of influenza and the benefits of the vaccine is needed. 
In particular, the problem of influenza being a highly contagious infection that can have serious complications (including death) needs to be presented to HCWs to help raise awareness of their responsibility to their patients. It should be emphasised that the risk of infection is higher in hospitals and other healthcare facilities, as should awareness and understanding by HCWs of the increased likelihood of disease transmission to their patients as well as to their close family and friends. The safety profile of the vaccine against seasonal influenza, which is based on many years of experience and many millions of doses administered each season with few serious side-effects, should be stressed. To further improve vaccination uptake by HCWs, such information should be coupled with an invitation to be vaccinated free of charge at a convenient time and venue, to be protected, and to protect their patients, family and friends.
Mandatory vaccination programmes are perceived very badly in the EU [8]. Their use could be counter-productive, probably resulting in higher levels of rejection of vaccination. Figure 1 presents the positive impact of increasing vaccination rates for HCWs for reducing influenza-related absenteeism, illness, and mortality. Simple presentation of such data, or evidence that for every $15 \mathrm{HCWs}$ who receive influenza vaccination one fewer person in the community contracts an influenza-like illness, can be used to stress the tangible benefits of HCW vaccination [25].

Table 2 presents options that could be implemented to promote vaccine uptake in hospital and practice settings. In particular, a dedicated team can oversee the vaccination programme to: deliver training; ensure an appropriate supply of vaccine; provide information; follow-up HCWs who remain unvaccinated. In addition, more personal approaches

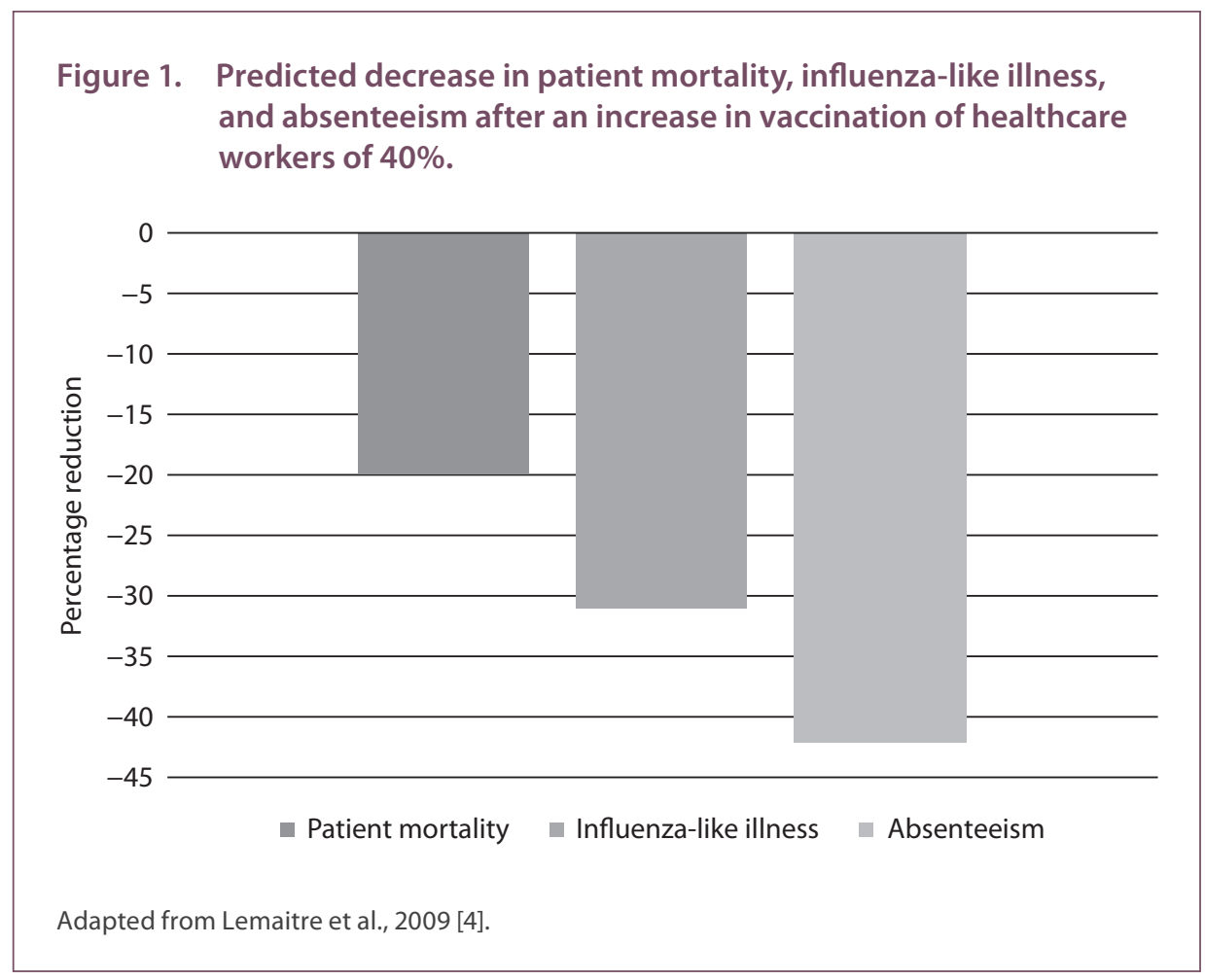

Table 2. Factors that could improve and promote uptake of influenza vaccination in hospital and practice settings.

- Appoint a dedicated team/individual with responsibility and accountability for influenza vaccination and associated issues

- Develop training and awareness programmes built around the risks of influenza and the benefits of vaccination, as well as the recommendations, benefits, and vaccines available

- Make an accurate assessment of the number of doses needed to vaccinate the workforce, and ensure timely delivery for vaccination sessions

- Publicise the dates for vaccination sessions widely throughout the hospital/practice

- Communicate with each HCW regarding logistics

- Audit vaccination rates throughout the season and send reminders to those HCWs who remain unvaccinated 
to physicians could be effective in increasing vaccination rates, but the message should be kept short, simple and clear.

\section{Discussion and future perspectives}

Addressing the problem of low rates of vaccination against seasonal influenza among HCWs in the EU through better education and increasing awareness regarding the benefits of vaccination will help to reduce the public-health and economic burden of seasonal influenza and reduce the number of deaths that result from complications of influenza infection in at-risk populations. The ethical challenge of not undertaking vaccination against a vaccine-preventable disease and the professional responsibility of HCWs to be vaccinated needs to be brought to the attention of HCWs. Some barriers to vaccination among HCWs will remain and a move towards compulsory vaccination might cause further alienation. However, offering a choice of other measures to limit the spread of influenza, such as mandatory use of facemasks at all times by HCWs who come into direct contact with patients, could be an option if vaccination is refused.

In the EU, vaccination against seasonal influenza is, in general, undervalued and there is scope to further emphasise its benefits. Such emphasis should be not only in relation to the professional responsibility of HCWs to be vaccinated but also using the personal benefits of being protected and helping protect their family and friends as important secondary drivers. Creating a better-vaccinated population of HCWs is likely to result in better HCW advocacy of vaccination against seasonal influenza. This in turn would be expected to result in better vaccination uptake in the population as a whole.

Such awareness can be achieved using a combination of tailored communication channels such as: personal contact; advocacy; posters; leaflets; emails; advertising on intranet sites, professional media and social media. In this way, a more informative message than 'the vaccine has arrived' can be conveyed. The HCWs' responsibility to be vaccinated, the vaccine being safe and effective, and the severity of influenza, should all be emphasised, together with an invitation to be vaccinated free of charge at a convenient time and location, to protect themselves, their patients, family and friends.

Use of case studies illustrating the feasibility of programmes and to demonstrate successes is a powerful source of inspiration. Successful policies regarding influenza vaccination incorporating such aspects (e.g., in the UK and the Netherlands) have led to an improvement in vaccination rates [6].

Furthermore, formalising the framework of a HCW's responsibility to be immunised against common serious communicable diseases (unless contraindicated), such as that outlined by the General Medical Council in the UK, who highlight HCW vaccination as Good Medical Practice, can be a motivating force [26]. In this context, as has been implemented in certain parts of the UK, a letter could be sent to HCWs at the start of each influenza season to improve implementation of a vaccination campaign and increase rates of vaccine coverage.

Access to vaccination needs to be made more straightforward for HCWs. Better infrastructure will help to convey the acceptance of influenza vaccination through investment in a robust programme of vaccination clinics. If HCWs feel vaccination to be inconvenient and time-consuming, improvements can be made in the efficiency in which the vaccination service is delivered. Well-organised systems such as dedicated vaccination clinics in which vaccination can be provided free of charge by dedicated occupational health practitioners will go some way to recognising these concerns and increasing vaccination rates for $\mathrm{HCWs}$.

\section{Conclusions}

Awareness of the potential for serious complications of influenza infection in pre-defined populations is low among $\mathrm{HCWs}$, as is their uptake of vaccination against seasonal influenza. Increasing this awareness is important, and can be done by highlighting the fact that HCWs are at risk of being infected and of subsequently transmitting the infection to vulnerable patients. HCWs need to lead by example from an informed position, and in this way not only improve vaccination rates for HCWs but also those of patients, to whom HCWs should act as advocates of vaccination against influenza.

\section{Acknowledgements and Contributions}

The author thanks Dr Andrew Lane (Lane Medical Writing), supported by Sanofi Pasteur MSD, for assistance in the preparation and development of this article in accordance with guidelines set by the European Medical Writers Association and Good Publication Practice.

\section{Potential conflicts of interest}

The International Committee of Medical Journal Editors' (ICMJE) Potential Conflicts of Interests form for the author is available for download at: http://www.drugsincontext.com/wp-content/ uploads/2015/1/dic.212268-COI.pdf

\section{Funding declaration}

This article was supported by Sanofi Pasteur MSD, Lyon, France. The author has received payments from Sanofi Pasteur MSD for lectures and advisory boards. However, no payment was made to the author for work on this article. 


\section{References}

1. Council of the European Union. State of play on implementation of the Council Recommendation of 22 December 2009 on seasonal influenza vaccination (2009/1019/EU). Available at http://ec.europa.eu/health/vaccination/docs/seasonflu_ staffwd2014_en.pdf [Last accessed: 18 December 2014].

2. Commission of the European Communities. Proposal for a Council Recommendation on Seasonal Influenza Vaccination 2009. Available at

http://ec.europa.eu/health/ph_threats/com/Influenza/ docs/seasonflu_rec2009_en.pdf [Last accessed: 18 December 2014].

3. Hayward AC, Harling R, Wetten S, Johnson AM, Munro S, Smedley J et al. Effectiveness of an influenza vaccine programme for care home staff to prevent death, morbidity, and health service use among residents: cluster randomised controlled trial. BMJ 2006;333(7581):1241. http://dx.doi.org/10.1136/bmj.39010.581354.55

4. Lemaitre $M$, Meret $T$, Rothan-Tondeur $M$, Belmin J, Lejonc $J L$, Luquel $L$ et al. Effect of influenza vaccination of nursing home staff on mortality of residents: a cluster-randomized trial. J Am Geriatr Soc 2009;57(9):1580-6. http://dx.doi.org/10.1111/j.1532-5415.2009.02402.x

5. Bonaccorsi G, Lorini C, Santomauro F, Guarducci S, Pellegrino E, Puggelli F et al. Predictive factors associated with the acceptance of pandemic and seasonal influenza vaccination in health care workers and students in Tuscany, Central Italy. Hum Vaccin Immunother 2013;9(12):2603-12. http://dx.doi.org/10.4161/hv.26036

6. Blank PR, Schwenkglenks M, Szucs TD. Vaccination coverage rates in eleven European countries during two consecutive influenza seasons. J Infect 2009; 58(6):446-58.

http://dx.doi.org/10.1016/j.jinf.2009.04.001

7. England $\mathrm{PH}$. Seasonal flu vaccine uptake (frontline healthcare workers - all trusts) 2013/2014 2014. Available at https://www.gov.uk/government/uploads/system/uploads/ attachment_data/file/274506/SeasonalFlu_UptakeDataHCWs_Dec13_acc.pdf [Last accessed: 18 December 2014].

8. Lukas K. Factors influencing healthcare professionals' willingness to be vaccinated. European Union Geriatric Medicine Society, Rotterdam, Netherlands, 17-19 September, 2014 [abstract/poster].

9. European Center for Disease Protection and Control. Seasonal influenza - basic facts. Available at http://ecdc.europa.eu/EN/HEALTHTOPICS/SEASONAL_ INFLUENZA/basic_facts/pages/basic_facts.aspx [Last accessed: 18 December 2014].

10. Blank P, Schwenkglenks M, Szucs TD. The impact of European vaccination policies on seasonal influenza vaccination coverage rates in the elderly. Hum Vaccin Immunother 2012;8(3):328-35.

http://dx.doi.org/10.4161/hv.18629
11. Centers for Disease Control and Prevention. Seasonal influenza: key facts about influenza (flu) and flu vaccine 2013. Available at

http://www.cdc.gov/flu/keyfacts.htm [Last accessed: 18 December 2014].

12. Vanhems $P$, Voirin $N$, Roche $S$, Escuret V, Regis $C$, Gorain $C$ et al. Risk of influenza-like illness in an acute health care setting during community influenza epidemics in 2004-2005, 2005-2006, and 2006-2007: a prospective study. Arch Intern Med 2011;171(2):151-7. http://dx.doi.org/10.1001/archinternmed.2010.500

13. WHO. Influenza: vaccine use. Available at http://who.int/influenza/vaccines/use/en/ [Last accessed: 18 December 2014].

14. Wilde JA, McMillan JA, Serwint J, Butta J, O'Riordan $M A$, Steinhoff MC. Effectiveness of influenza vaccine in health care professionals: a randomized trial. JAMA 1999;281(10):908-13. http://dx.doi.org/10.1001/jama.281.10.908

15. Potter J, Stott DJ, Roberts MA, Elder AG, O'Donnell B, Knight $P V$ et al. Influenza vaccination of health care workers in long-term-care hospitals reduces the mortality of elderly patients. J Infect Dis 1997;175(1):1-6. http://dx.doi.org/10.1093/infdis/175.1.1

16. Preaud E, Durand L, Macabeo B, Farkas N, Sloesen B, Palache $A$ et al. Annual public health and economic benefits of seasonal influenza vaccination: a European estimate. BMC Public Health 2014;14:813. http://dx.doi.org/10.1186/1471-2458-14-813

17. Centers for Disease Control and Prevention. Seasonal influenza (flu) 2013. Available at http://www.cdc.gov/flu/about/qa/disease.htm [Last accessed: 18 December 2014].

18. WHO. Vaccines against influenza WHO position paper - November 2012. Releve epidemiologique hebdomadaire / Section d'hygiene du Secretariat de la Societe des Nations = Weekly epidemiological record / Health Section of the Secretariat of the League of Nations. 2012;87(47):461-76.

19. European Centre for Disease Prevention and Control. Revised estimates of deaths associated with seasonal influenza in the US 2010. Available at http://www.ecdc.europa.eu/en/activities/sciadvice/_ layouts/forms/Review_DispForm.aspx?List=a3216f4cf040-4f51-9f77-a96046dbfd72\&ID=394 [Last accessed: 18 December 2014].

20. Kuster SP, Shah PS, Coleman BL, Lam PP, Tong A, Wormsbecker $A$ et al. Incidence of influenza in healthy adults and healthcare workers: a systematic review and meta-analysis. PloS One 2011;6(10):e26239. http://dx.doi.org/10.1371/journal.pone.0026239

21. National Institue for Health and Clinical Excellence. Available at http://guidance.nice.org.uk/page.aspx?o=440453 [Last accessed: 18 December 2014]. 
22. European Centre for Disease Prevention and Control. New and updated evaluations of neuraminidase inhibitors for preventing and treating influenza published 2014. Available at

http://ecdc.europa.eu/en/activities/sciadvice/_layouts/ forms/Review_DispForm.aspx?List=a3216f4c-f0404f51-9f77-a96046dbfd72\&ID=764 [Last accessed: 18 December 2014].

23. European Centre for Disease Protection and Control. Multimedia: influenza videos. Available at http://ecdc.europa.eu/EN/HEALTHTOPICS/SEASONAL INFLUENZA/multimedia_tools/pages/

multimedia_tools.aspx

(accessed 18 December 2014).
24. National Institue for Health and Clinical Excellence. Oseltamivir, amantadine (review) and zanamivir for the prophylaxis of influenza 2008. Available at http://www.nice.org.uk/guidance/TA158/chapter/ 1-guidance [Last accessed: 18 December 2014].

25. Press $M$. When hospital workers get vaccines, community flu rates fall (2014). Available at http://medicalxpress.com/news/2014-06-hospital-workersvaccines-flu-fall.html [Last accessed: 18 December 2014].

26. General Medical Council. Good Medical Practice 2013. Available at http://www.gmc-uk.org/static/documents/content/Good_ medical_practice_-_English_0914.pdf [Last accessed: 14 January 2015]. 